\title{
Survival after Metastasectomy for Metastatic Urothelial Carcinoma: A Systematic Review and Meta-Analysis
}

\author{
Vaibhav Patel ${ }^{\mathrm{a}, 1, *}$, Ana Collazo Lorduy ${ }^{\mathrm{b}, \mathrm{c}, 1}$, Aaron Stern ${ }^{\mathrm{a}}$, Omar Fahmy ${ }^{\mathrm{d}}$, Rachel Pinotti ${ }^{\mathrm{e}}$, \\ Matthew D. Galsky ${ }^{\mathrm{f}}$ and Georgios Gakis ${ }^{\mathrm{g}}$ \\ ${ }^{a}$ Icahn School of Medicine at Mount Sinai, New York, NY, USA \\ ${ }^{\mathrm{b}}$ Department of Pathology, Icahn School of Medicine at Mount Sinai, New York, NY, USA \\ ${ }^{c}$ Spanish Society of Medical Oncology, Madrid, Spain \\ ${ }^{\mathrm{d}}$ Department of Urology, Universiti Putra Malaysia, Selangor, Malaysia \\ ${ }^{\mathrm{e}}$ Information and Education Service, Icahn School of Medicine at Mount Sinai, New York, NY, USA \\ ${ }^{\mathrm{f}}$ Icahn School of Medicine at Mount Sinai, Division of Hematology and Medical Oncology, Tisch Cancer Institute, \\ New York, NY, USA \\ ${ }^{\mathrm{g}}$ Urology and Nephrology Center, University of Mansoura, Mansoura, Egypt
}

\begin{abstract}
.
Background: Cisplatin-based combination chemotherapy is standard treatment for metastatic urothelial carcinoma; however, the vast majority of patients experience disease progression. As systemic therapy alone is rarely curative for the treatment of metastatic urothelial cancer, not only are new therapies needed but also refinement of general treatment principles. Herein, we conducted a systematic review and meta-analysis to explore the role of metastasectomy in metastatic urothelial carcinoma. Methods: We conducted a systematic review of the literature regarding local treatment for metastatic urothelial carcinoma. An online electronic search of the PubMed/MEDLINE and EMBASE databases was performed to identify peer-reviewed articles. All procedures were performed according to the Preferred Reporting Items for Systematic Reviews and MetaAnalyses (PRISMA) guidelines. Information was then extracted including number of patients, gender, the site of the primary urothelial tumor, site of metastasis, chemotherapy before or after metastasectomy, overall survival (OS), and disease specific survival (DSS) after metastasectomy. A meta-analysis was performed with those studies with sufficient survival data to obtain pooled overall survival. The article quality was assessed using the Cochrane Handbook "risk of bias" tool.

Results: Seventeen out of 3963 articles were eligible for review between 1990-2015, including a total of 412 patients. The mean time to recurrence after metastasectomy was 14.25 months. The overall survival from time of metastasectomy ranged from 2 to 60 months. Pooled analyses of studies reported survival data revealed an improved overall survival for patients treated with metastasectomy compared with non-surgical treatment of metastatic lesions (HR 0.63; 95\% CI, 0.49-0.81). All, except for three studies, were retrospective and non-randomized, leading to a high risk of bias associated with patient selection, patient attrition, and reporting. Such high potential of selection bias may lead to higher OS than expected. Additionally, treatment and outcome details reported across studies was highly variable.

Conclusions: Limited conclusions can be drawn from the available literature exploring the role of metastasectomy in the management of metastatic urothelial cancer due to lack of uniform reporting elements and multiple sources of bias particularly related to a lack of prospective randomized trials. As a subset of patients treated with metastasectomy achieve durable disease control, this approach may be considered for select patients.
\end{abstract}

Keywords: Urothelial carcinoma, bladder cancer, metastasectomy, transitional cell carcinoma

\footnotetext{
${ }^{1}$ Both authors contributed equally to this article.

*Correspondence to: Vaibhav Patel, Department of Medicine, Icahn School of Medicine at Mount Sinai, 1 Gustave Library Pl,
}

New York, NY 10029, USA. Tel.: +1 224578 0916; E-mail: vaibhav.patel@mountsinai.org. 


\section{BACKGROUND}

Cisplatin-based combination chemotherapy is standard treatment for patients with metastatic urothelial cancer. While the majority of patients respond to such treatment, and complete clinical responses are achieved in approximately $10-20 \%$ of patients, the vast majority of patients experience disease progression and subsequently succumb to the disease $[1,2]$. After several decades without improvements in the outcomes of patients with metastatic urothelial cancer [3], immune checkpoint blockade has recently been shown to achieve durable responses and improve survival in patients with metastatic urothelial cancer [4-6]. However, only a small subset of patients with metastatic urothelial cancer respond to systemic or immunomodulator therapy, treatment is generally not curative, and alternative therapeutic strategies are thus needed to achieve durable disease control [7-9].

Post-chemotherapy resection of residual disease is a well-defined strategy for the treatment of other solid tumors such as testicular germ cell tumors [10-13]. A possible rationale for post-chemotherapy surgery for residual urothelial cancer was initially proposed by Dimopoulos et al. in 1994 in an analysis demonstrating a high incidence of progression at prior sites of disease in patients who had initially responded to systemic chemotherapy [14]. Since that time, there have been numerous predominantly single institutional series attempting to define the optimal use of post-chemotherapy surgery for residual disease in urothelial cancer. Herein, we systematically review the available literature regarding the role of metastasectomy in metastatic urothelial cancer, which included both synchronous and metachronous metastases at presentation, and perform a metaanalysis to refine the role of this multimodality approach.

\section{MATERIALS AND METHODS}

\section{Search strategy}

We conducted a systematic search according to the PRISMA guidelines [15], and in accordance with the principles outlined in the Cochrane Handbook for Systematic Reviews of Interventions [16]. The PubMed/MEDLINE and EMBASE databases were systematically searched for studies between 1990-2015 reporting on local treatments for metastatic urothelial carcinoma. The comprehensive search strategy included both subject headings and a comprehensive list of keywords as outlined in Appendix 1. Animal studies were excluded using the method recommended by the Cochrane Handbook for Systematic Reviews of Interventions [16]. The full search strategy for both databases is reported in Appendix 1.

\section{Study selection}

The study selection process is outlined in Fig. 1. Three reviewers, which include the first three authors of this manuscript, independently screened titles and abstracts and the following studies were excluded after the initial literature search: reviews, case reports, studies not reported in English language, studies of systemic treatment only, studies of localized disease only, and studies of other genitourinary tumors. Any disagreement was resolved by consensus among the reviewers. The study population included those patients with metastatic urothelial carcinoma to any organ, except for regional pelvic lymph nodes, as this population is the topic of another study. There were no specific restrictions on prior treatment including surgery, radiotherapy, and chemotherapy. After removing duplicates, 3744 records were screened by title of which 3024 were excluded. Out of the remaining 720 articles screened by abstract, 665 were excluded. A final list of 55 manuscripts were read in full to determine eligibility.

\section{Data extraction}

Initially, data was extracted independently by three authors. The following variables were extracted: number of patients, gender, the site of the primary urothelial tumor, site of metastasis, chemotherapy before or after metastasectomy, overall survival (OS), progression-free survival (PFS), and disease specific survival (DSS) after metastasectomy. Hazard ration (HR) and its 95\% CI were extracted from KaplanMeier graphs using Tierney's method [17]. In one study without a Kaplan-Meier curve [18], the graph was drawn first based on the reported individual data of the patients in this study; then Tierney's method was applied.

\section{Outcome measures}

The primary outcomes assessed was OS, while DSS and PFS were secondary outcomes. 


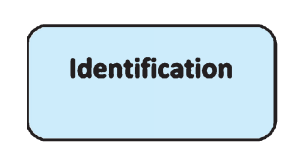

\section{Screening}

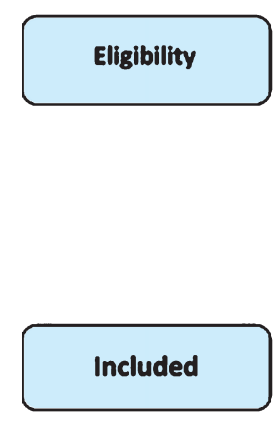

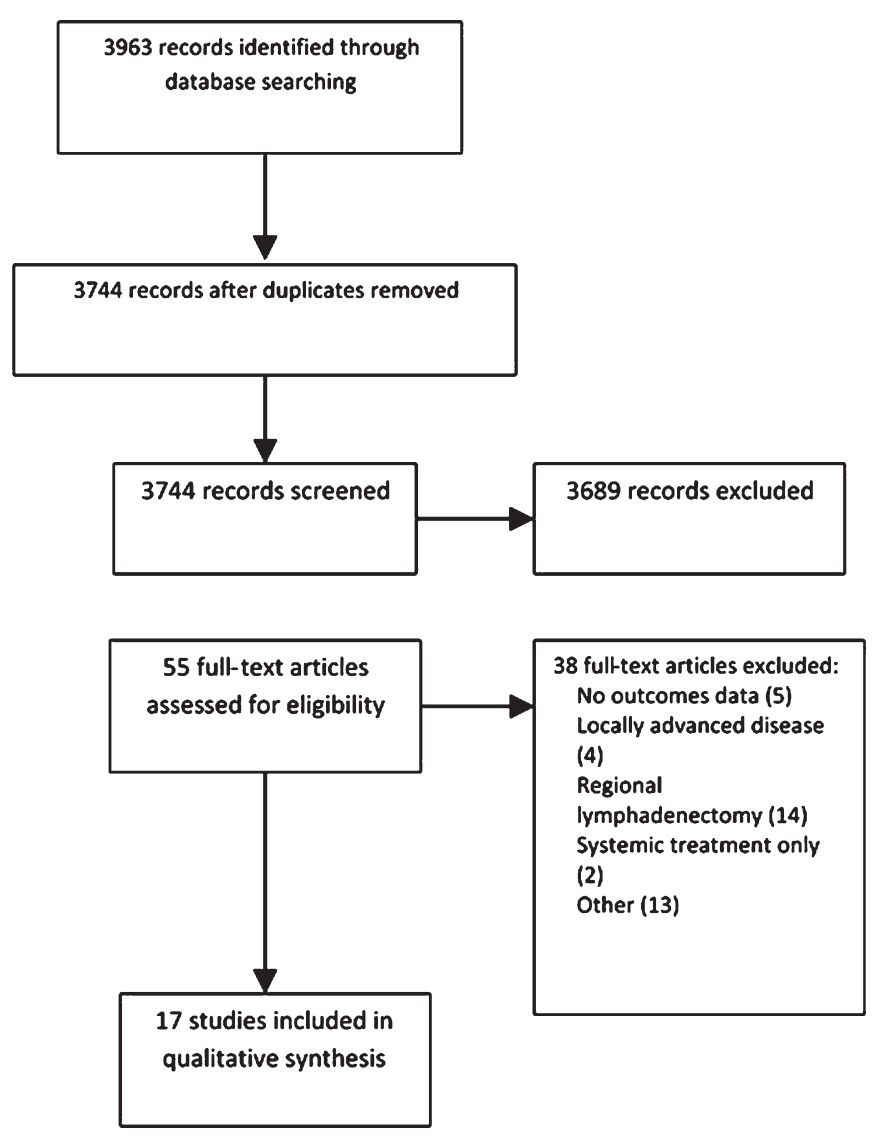

Fig. 1. Flow diagram following PRISMA statement.
Descriptive statistics were used to summarize baseline characteristics.

\section{Statistical analysis}

The meta-analysis was conducted using Review Manager (RevMan) software version 5.3 (The Nordic Cochrane Centre, The Cochrane Collaboration, Copenhagen). A random effect model was applied regardless of $\mathrm{I}^{2}$ value due to the expected heterogeneity between the included studies. The analysis of generic inverse variance was applied using Log HR and its standard error (SE).

\section{Quality assessment}

The Cochrane Handbook "risk of bias" tool was utilized for risk of bias assessment for each of the studies of interest [16]. Risk of bias was stratified by score as follows: $1=$ low, $2=$ unclear, $3=$ high. Evidence quality was not assessed by GRADE because of the nature of the included studies (ie, retrospective, non-comparative studies), and the high risk of bias across the studies.

\section{RESULTS}

\section{Search results}

Our literature search identified 3963 articles, 55 of which were selected for full text screening. Among the 55 articles, 17 articles on 412 patients met criteria for inclusion in the final analysis (Fig. 1) including 15 retrospective studies and 2 prospective studies $[19,20]$. Six studies included metastasectomy at multiple metastatic sites [20-26], three studies focused on the role of distant lymphadenectomy $[19,27,28]$, five involved pulmonary metastasectomy [26, 29-32], and three reported resection of brain metastases [18, 33, 34]. The details of these studies are outlined in Table 1. 


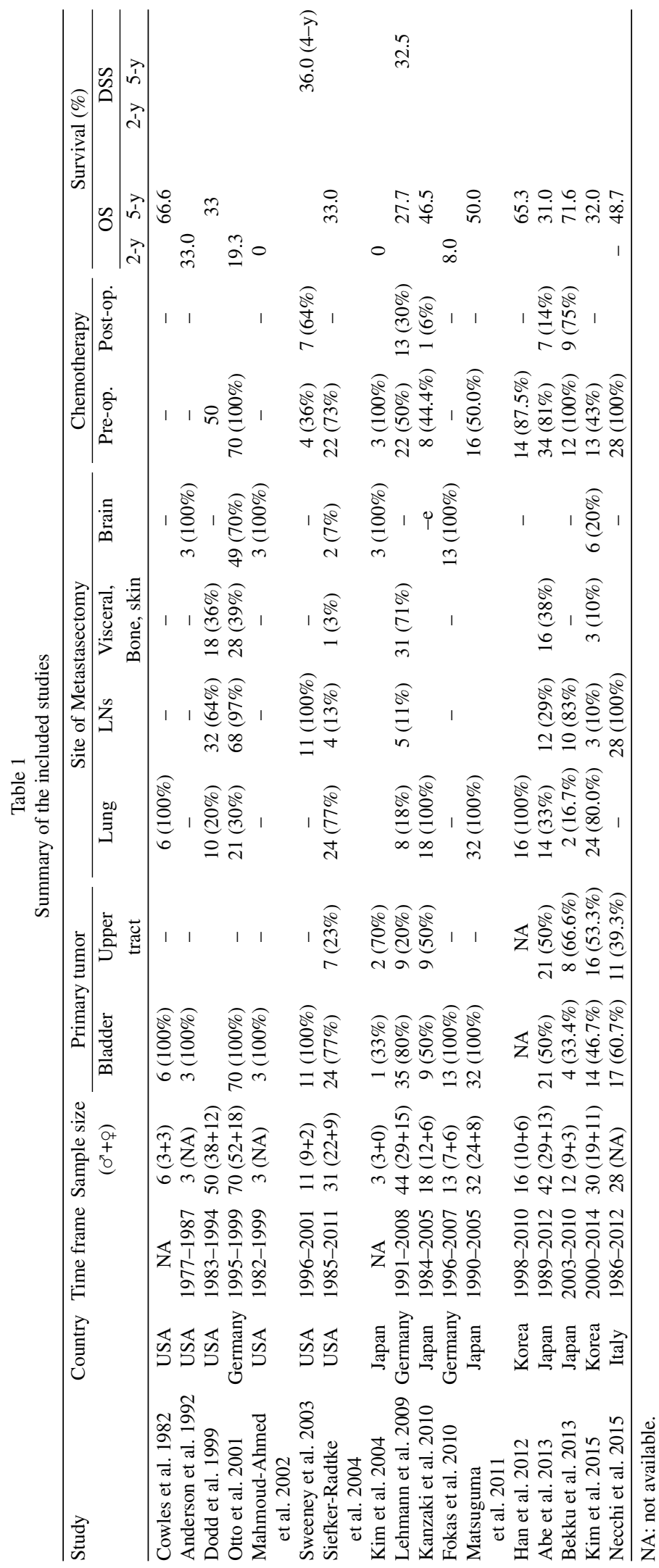


Table 2

Pooled patient characteristics across 17 included studies

\begin{tabular}{|c|c|}
\hline \multicolumn{2}{|l|}{ Type of study } \\
\hline Retrospective cohort & 14 \\
\hline Randomized controlled trial & 3 \\
\hline Total number of patients & 412 \\
\hline \multicolumn{2}{|l|}{ Demographics, N (\%) } \\
\hline Male & $288(70)$ \\
\hline Mean age & 62 \\
\hline \multicolumn{2}{|l|}{ Tumor location, $\mathrm{N}(\%)$} \\
\hline Bladder & $309(75)$ \\
\hline Other ${ }^{\mathrm{a}}$ & $103(25)$ \\
\hline \multicolumn{2}{|l|}{ Number of metastases, $\mathrm{N}(\%)$} \\
\hline Multiple & $148(36)$ \\
\hline Single & $148(36)$ \\
\hline Not reported & $116(28)$ \\
\hline \multicolumn{2}{|l|}{ Sites of metastasectomy, $\mathrm{N}$} \\
\hline Lung & 181 \\
\hline Bone & 21 \\
\hline Liver & 16 \\
\hline Distant lymph nodes & 118 \\
\hline Other & 47 \\
\hline \multicolumn{2}{|l|}{ Peri-operative chemothereapy, N (\%) } \\
\hline Yes & $325(79)$ \\
\hline No & $66(16)$ \\
\hline Not reported & $21(5)$ \\
\hline \multicolumn{2}{|l|}{ Type of chemotherapy, N (\%) } \\
\hline Platinum-based chemotherapy & $304(88)$ \\
\hline Other & $21(6)$ \\
\hline Unknown & $21(6)$ \\
\hline \multicolumn{2}{|l|}{ Outcomes } \\
\hline \multicolumn{2}{|c|}{ Mean time from initial surgery to metastasectomy ${ }^{\mathrm{b}} 19$ months } \\
\hline Mean time for relapse after metastasectomy ${ }^{\mathrm{c}}$ & 14.3 month \\
\hline
\end{tabular}

\section{Characteristics for reviewed studies}

Among the included articles, there was marked variability in the reporting of patient characteristics and clinical outcomes, systemic treatment strategy, and in the extent of metastasectomy.

The characteristics of the 412 patients included in the 17 articles are detailed in Table 2 . The mean age of the patients was 62 years, and $70 \%$ were male. Most patients had bladder primary tumors, although $25 \%$ of patients had tumors in the upper urinary tract or urethra. The number of metastases was reported in $72 \%$ of patients and $50 \%$ of these patients had multiple metastases. The mean time from the initial surgery, either transurethral resection of the bladder tumor (TURBT) or cystectomy, to metastasectomy was 19 months, although this data was only reported in 6 studies [20, 21, 23, 28, 30]. The sites of metastasectomy are outlined in Table 1 with resection of lung metastases representing the most common site.
Systemic treatment generally consisted of chemotherapy given before and/or after metastasectomy. Two studies did not specify the treatment schedule [18, 32]. Only 9 articles specified the type of systemic treatment employed [18-20, 24, 27, 29, 30, 32, 34]; the combination of methotrexate, vinblastine, doxorubicin, plus cisplatin (MVAC) was the most commonly used chemotherapy regimen.

\section{Studies limited to patients with lung metastases}

Five retrospective studies evaluated the role of lung metastasectomy in patients with metastatic bladder cancer [26, 29-32] as detailed in Fig. 2. These studies, including 96 patients, reported 5-year OS ranging from $46.5 \%$ to $85.3 \%$ except for one study which only reported 3-year OS (41\%) [29]. Only two of the studies reported 5-year PFS [26, 31], which ranged from $26 \%$ to $50 \%$. Several studies attempted to find co-variates that could predict good outcomes. Two studies reported that solitary metastases were independently associated with better prognosis $[30,32]$ with 5-year OS ranging from $83.3 \%$ (HR not reported) to $85.7 \%$ (HR not reported, $p=0.009$ ). Another study [29] showed a much longer PFS (68 months vs. 7 months, $p<0.001)$ in the group with solitary metastasis when compared to those patients with multiple metastatic pulmonary lesions. Furthermore, one study [26] also showed that solitary metastases $<3 \mathrm{~cm}$ (HR 4.72, $p=0.006$ ) were associated with improved outcomes compared with patients with larger metastases.

All the studies, except one [29], included cases with only pulmonary metastases (and not other sites) amenable to resection. Between these different studies, there were no uniform guidelines regarding number of times metastectomy can be performed and use of chemotherapy in the post-metastectomy setting. For example in some of the studies [26, 29, 31], patients underwent an aggressive surgical approach with serial metastasectomies performed for recurrent tumors.

\section{Studies limited to patients with distant lymph node metastases}

Three studies specifically addressed the role of retroperitoneal lymph node dissection after chemotherapy for the management of metastatic bladder carcinoma [19, 27, 28] as shown in Fig. 2. Two retrospective studies with a total of 31 patients [27, 28] and one phase II trial [19] with 11 patients, 


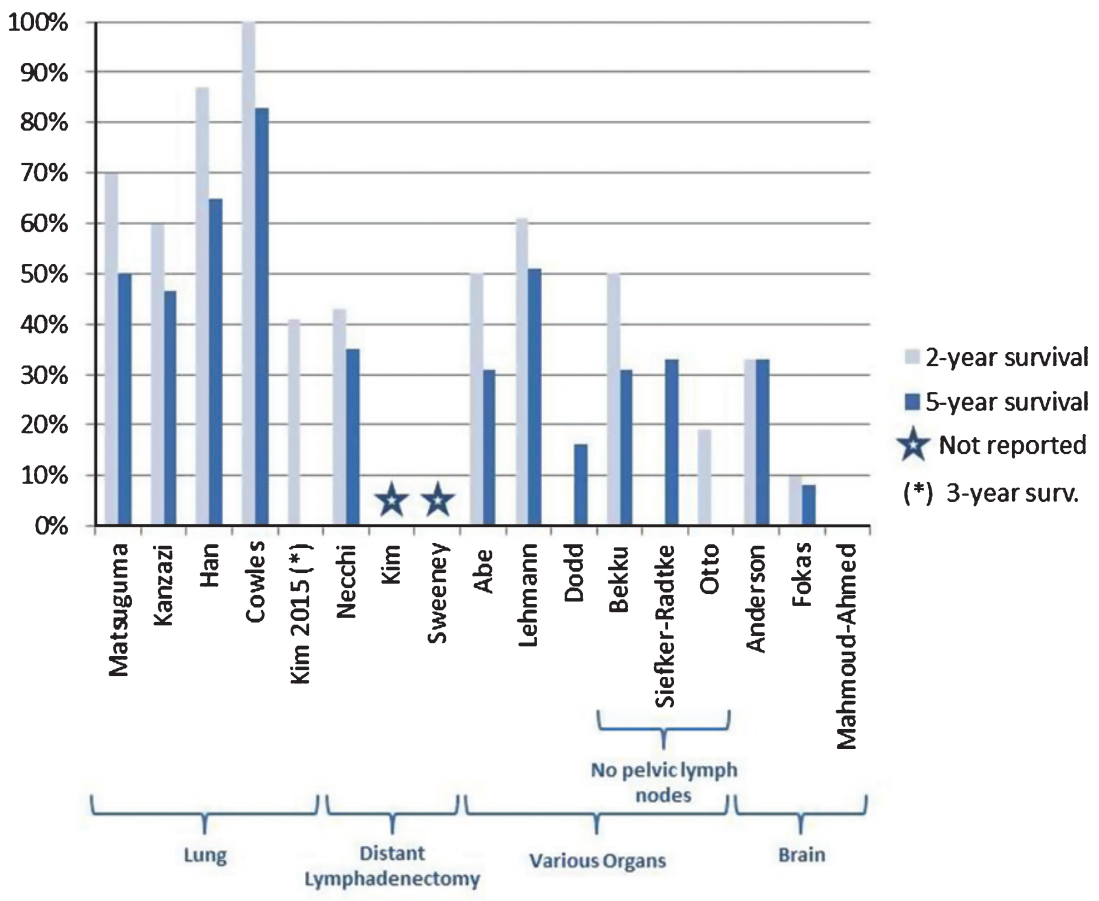

Fig. 2. 2 and 5-year overall survival reported across 17 studies included in the analysis.

showed median PFS and OS ranging between 7-20 months and 8-37 months, respectively.

Necchi et al. retrospectively studied 28 patients over a 26-year period, while Sweeney prospectively enrolled 11 patients. Kim et al. presented a small case series consisting of only three patients. All the studies were limited to patients with nodal metastasis and at least stable disease after platinum-based chemotherapy. The study by Necchi et al. included patients with retroperitoneal nodal metastasis and those with pelvic nodal recurrence, while the other two studies only included patients with distant nodal involvement. Two of the studies $[19,28]$ only included patients achieving a partial response (PR) or complete response (CR) to systemic chemotherapy, while the study reported by Necchi et al. also included patients with stable disease. Interestingly, the study by Necchi et al. reported an analysis between the group of patients with $\mathrm{CR}$ to chemotherapy, those with $\mathrm{CR}$ after removal of residual nodal disease, and those with residual disease, and found a median OS to be 44 months, 37 months, and 12 months, respectively. In the study by Sweeney et al., among patients with a clinical CR with chemotherapy, 6 of 7 patients had pathologic evidence of residual cancer in the lymph nodes. On multivariate analyses, patients with viable tumor in no more than 2 lymph nodes correlated with significantly greater PFS (5 months vs. median not achieved at 60 months, $p=0.01$ ) and DSS ( $p=8$ months vs. median not achieved at 60 months, $p=0.006$ ).

\section{Studies limited to brain metastases}

Three retrospective studies [18, 33, 34], consisting of 87 patients, studied the role of resection of brain metastases with median OS for all patients ranging from 2 to 29 months. There was a large variation in the extent of metastases and the use of multi-modal treatment strategies consisting of either whole-brain radiation therapy (WBRT), surgery with WBRT, and stereotactic radiosurgery (SRS) with or without WBRT. In all three studies, WBRT was the predominant treatment for multiple brain lesions in the setting of uncontrolled systemic disease, while patients underwent combined surgical resection plus WBRT for solitary lesions. SRS was reserved for lesions that were $<3 \mathrm{~cm}$. The median OS for patients treated with WBRT only and surgical resection + WBRT ranged between 1.4-2 months and 7.8-29 months, respectively. Fokas et al. also performed multivariate analyses and found that a lack of extracerebral metastases was a favorable prognostic factor (RR: 7.45; 95\% CI: 2.27-24.41; $p=0.001$ ) 


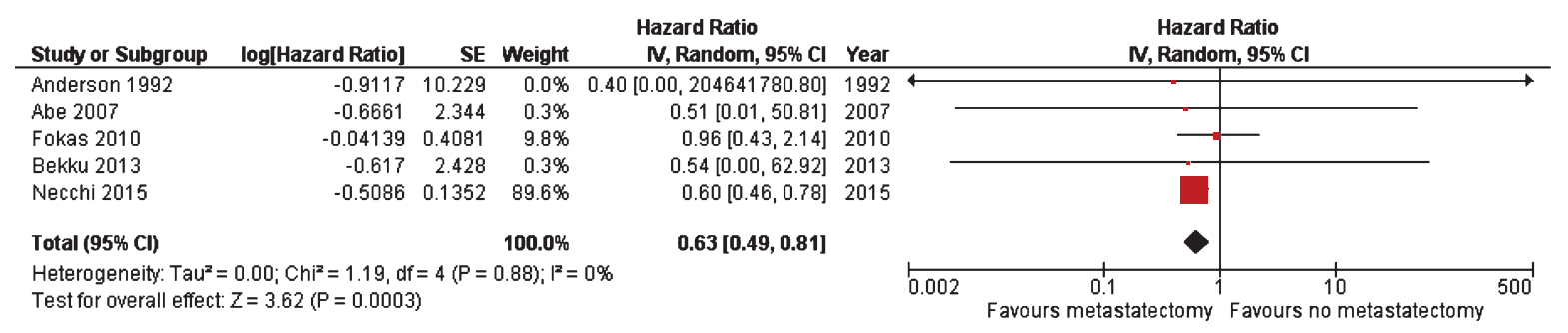

Fig. 3. Meta-analysis of OS among five studies where survival measures could be extracted or calculated from Kaplan-Meier curves.

in those receiving WBRT and surgical resection plus WBRT [33].

\section{Studies including other metastatic sites}

Six studies included patients with distant metastases in various anatomic locations that underwent complete resection [20-25]. The 5-year median OS from metastasectomy ranged from $31 \%-71.6 \%$, while one study [20] reported a 2-year OS at $19.3 \%$.

All of these studies, with the exception of a prospective phase II trial, by Otto and colleagues [20], were retrospective in nature. Among the six studies, two were multi-center [21, 23] and the remainder involved are single-institutions. The locations of metastases were similar among studies, although some included pelvic lymph node resections $[21,23,24]$ in the metastasectomy group. All of the studies, with the exception of the prospective study [20], included patients with involvement of one anatomic site with one or multiple, small lesions. There was variability in the number of patients who received chemotherapy either before or after metastasectomy. Additionally, there was heterogeneity in the response to chemotherapy prior to metastasectomy; four studies [21, 23-25] included patients undergoing metastasectomy after achieving a CR to chemotherapy, one study [22] included only patients with PR to chemotherapy, and one study [20] included only patients that were refractory to chemotherapy. Interestingly in this study, those patients who underwent metastasectomy for palliative purposes had a significant improvement in quality-of-life (WHO performance score 3.3 to $2.1, p=0.005$ ). The pathologic evidence of residual disease in the metastasectomy specimen varied across studies. The study by Bekku et al. reported the longest 5-year PFS and OS at 63.9\% and $71.6 \%$, with viable tumor in only $25 \%$ of patients after chemotherapy, while the other studies reported pathologic evidence of residual tumor in $66-91 \%$ of patients.

\section{Meta-analysis}

The mean time to recurrence after metastasectomy was 14.3 months. However, this data was only reported in nine out of 17 studies [19, 23, 25, 26, 28-32]. The overall survival from the resection of metastases reported in the different articles ranged from 2 to 60 months.

A meta-analysis was performed on five studies where survival measures could be extracted or calculated from Kaplan-Meier curves. Metastasectomy displayed a significant better OS in comparison to non-surgical treatment of metastatic lesions (HR 0.63; 95\% CI, 0.49-0.81), as shown in Fig. 3. Of note, one [27] of the five studies was weighted $90 \%$ based on its sample size, and the final HR is largely reflective of this one study. Meta-analysis to compare the impact of location of metastasectomy on survival was unable to be performed due to a lack of uniform data.

\section{Risk of bias and confounding.}

Table 3 summarizes the risk of confounding and overall bias for the included studies in the systematic review. All, except for three studies, were retrospective and non-randomized, leading to the high risk of bias associated with patient selection, patient attrition, and selective reporting. Regarding confounders, all of the studies reported adequate data on age and gender. Reporting of systemic treatment type, treatment schedules, and response to treatment were heterogeneous. Information regarding performance was not available for most of the studies.

\section{DISCUSSION}

While metastasectomy plays a role in the management of select patients with metastatic urothelial cancer, the optimal application of such treatment remains elusive. Furthermore, outcomes of patients 


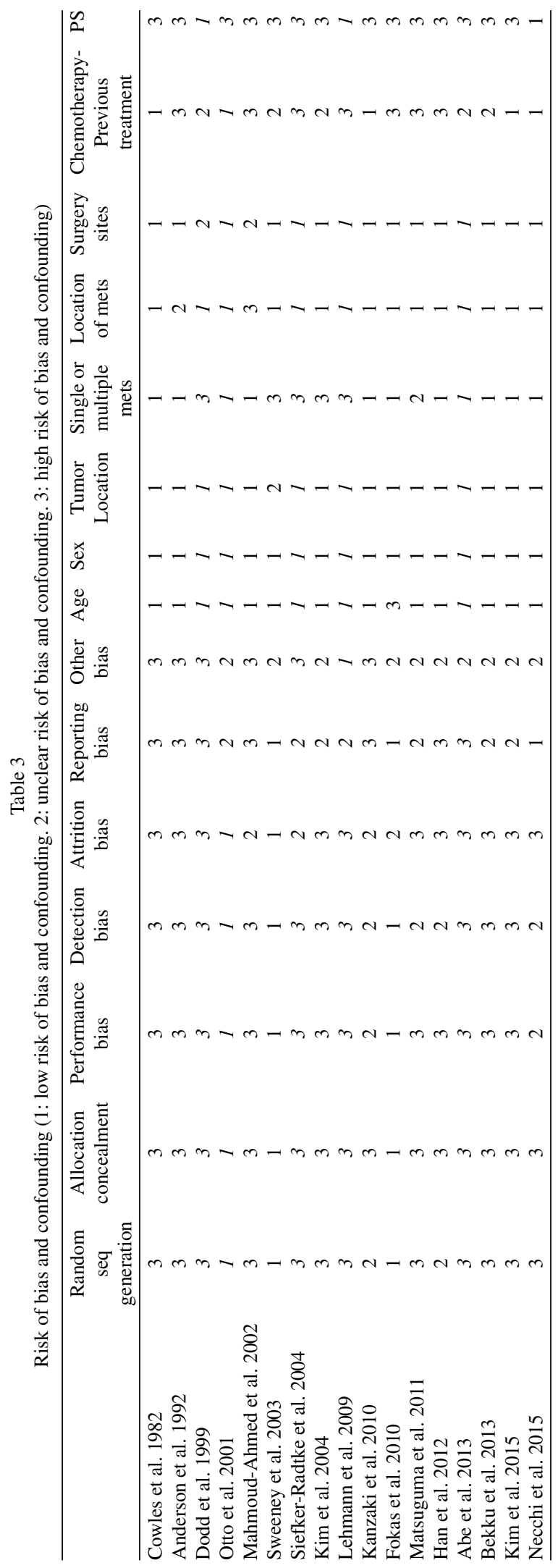


treated with metastasectomy are not well defined and are based on mostly small single-institutional studies. To better understand the state of knowledge regarding metastasectomy in metastatic urothelial cancer and to define knowledge gaps and opportunities for further study, we performed a systematic review and meta-analysis.

A major challenge we encountered when conducting this systematic review and meta-analysis was that the literature is mostly comprised of singleinstitution, small retrospective series that suffer markedly from selection bias. There is a lack of uniform reporting compromising the ability to pool data for analysis. This lack of uniform reporting ranged from the details and timing of systemic therapy, sequence of therapies, response to chemotherapy, presentation of synchronous or metachronous metastases, and surgical complications. Our meta-analysis of five studies with available comparative data revealed that patients undergoing metastasectomy displayed a significantly better OS in comparison to patients undergoing non-surgical treatment of metastatic lesions. However, a major limitation of the meta-analysis is that one study [27] contributed overwhelmingly to these results (i.e., was weighted $90 \%$ ). This study by Necchi included patients who had exclusive subdiaphragmatic, abdominal, or pelvic nodal disease, and had at least stable disease after receiving mMVAC. Of the 59 patients studied, 28 underwent pelvic or retroperitoneal lymphadenectomy. When compared to the 31 patients who did not undergo surgery, those patients that had removal of residual disease demonstrated a survival advantage. Despite the limitations outlined above, our meta-analysis revealed that patients undergoing metastasectomy displayed a significantly better OS in comparison to patients undergoing non-surgical treatment of metastatic lesions. Interpretation of these data are limited by marked selection bias in the included studies. Therefore, these studies, and our pooled data, demonstrate what is potentially achievable regarding patient outcomes, even in the context of metastatic urothelial cancer. Whether the patients achieving durable disease control post-metastasectomy would have fared equally well without a surgical approach cannot be answered definitively in the absence of randomized trials. While several barriers exist to the conduct of such a trial, both from a standpoint of trial design and feasibility, definitively addressing the role of this approach should be considered by the bladder cancer clinical trials community. In the absence of a prospective randomized trial, a prospective registry employing uniform data capture and follow-up across multiple sites of care could help refine the optimal use of metastasectomy.

In the absence of prospective data, and randomized trials, selecting appropriate patients for metastasectomy remains challenging. Given the lack of access to individual patient data, we were unable to dissect the baseline characteristics of patients who may derive the most benefit from metastasectomy. Several of the individual studies included in our systematic review attempted to define such factors. For example, in the studies by Matsugama [26] and Kanzazi et al. [30], pulmonary metastasectomy was purported to be most beneficial in patients with small, solitary pulmonary lesions. Response to initial chemotherapy was suggested as a strategy to triage patients to metastasectomy in other studies. In the study by Necchi et al., patients with at least stable disease could undergo surgery [9], while in the other two studies significant partial responses were required. Sites and number of metastases may play a key role in impacting outcomes. In several analyses, better outcomes were reported in patients with limited sites of metastases, mainly to the lymph nodes or to the lungs $[21,22,24,26,27,29]$. Not surprisingly, patients with solitary lesions appeared to also have a better prognosis [21]. Together, these studies suggest that low volume chemotherapy-sensitive metastatic disease confined to lungs or lymph nodes may be optimal for integrating metastasectomy; however, the extent and pace of disease in such patients may reflect favorable underlying disease biology and such patients may have theoretically have favorable outcomes even in the absence of metastasectomy and patient counseling regarding the limitations of the available data is required to facilitate shared medical decisions.

Importantly, metastectomy may not only play a role in improving survival but may also be considered for palliative reasons. Otto et al. conducted the only study that elucidated the role of metastectomy on quality of life in patients with disease not amenable to chemotherapy [20]. The study showed that surgical removal of metastases in such patients could favorably impact in quality of life in symptomatic patients but could worsen in asymptomatic patients without any survival advantage.

The landscape of treatment for metastatic urothelial cancer has markedly changed in recent years with the introduction of immune checkpoint blockade [35, 36]. A subset of patients experiences durable disease control with PD-1/PD-L1 blockade 
though most commonly without achieving a complete response. Further, a subset of patients treated with such therapies experience oligoprogression via asyet-undefined acquired resistance mechanisms. The role of metastasectomy may need to be completely re-envisioned as modulation of the host immune system is appearing to alter the course of disease, and patterns of progression, in ways that were uncommon in the era of cytotoxic chemotherapy. Whether or not immune checkpoint blockade plus metastasectomy will achieve cure is some patients with metastatic urothelial cancer is unclear but is ripe for investigation.

In conclusion, to our knowledge, this is the first systematic review and meta-analysis to identify the evidence-base regarding the role of metastasectomy in patients with urothelial carcinoma. Given the potential for durable disease control, metastasectomy should be considered as a therapeutic option in select patients. However, the current level of evidence precludes establishing more general recommendations for use of this approach.

\section{FUNDING}

This work has partially been funded by Spanish Society of Medical Oncology (ACL).

\section{CONFLICT OF INTEREST}

The authors have no conflict of interest to report.

\section{REFERENCES}

[1] Gallagher DJ, Milowsky MI, Bajorin DF. Advanced bladder cancer: Status of first-line chemotherapy and the search for active agents in the second-line setting. Cancer 2008;113(6):1284-93.

[2] Sternberg CN, Yagoda A, Scher HI, Watson RC, Geller $\mathrm{N}$, Herr HW, et al. Methotrexate, vinblastine, doxorubicin, and cisplatin for advanced transitional cell carcinoma of the urothelium. Efficacy and patterns of response and relapse. Cancer 1989;64(12):2448-58.

[3] Pal SK, Lin YI, Yuh B, DeWalt K, Kazarian A, Vogelzang N, et al. Conditional Survival in de novo Metastatic Urothelial Carcinoma. PLoS One 2015;10(8):e0136622.

[4] First-line atezolizumab effective in bladder cancer. Cancer Discovery 2016;6(8):Of7.

[5] Bellmunt J, de Wit R, Vaughn DJ, Fradet Y, Lee JL, Fong L, et al. Pembrolizumab as Second-Line Therapy for Advanced Urothelial Carcinoma. The New England Journal of Medicine 2017;376(11):1015-26.

[6] Sharma P, Retz M, Siefker-Radtke A, Baron A, Necchi A, Bedke J, et al. Nivolumab in metastatic urothelial carcinoma after platinum therapy (CheckMate 275): A multicentre, single-arm, phase 2 trial. The Lancet Oncology 2017;18(3):312-22.
[7] Sternberg CN, de Mulder PH, Schornagel JH, Theodore C, Fossa SD, van Oosterom AT, et al. Randomized phase III trial of high-dose-intensity methotrexate, vinblastine, doxorubicin, and cisplatin (MVAC) chemotherapy and recombinant human granulocyte colony-stimulating factor versus classic MVAC in advanced urothelial tract tumors: European Organization for Research and Treatment of Cancer Protocol no. 30924. Journal of Clinical Oncology: Official Journal of the American Society of Clinical Oncology 2001;19(10):2638-46.

[8] Bellmunt J, von der Maase H, Mead GM, Skoneczna I, De Santis M, Daugaard G, et al. Randomized phase III study comparing paclitaxel/cisplatin/gemcitabine and gemcitabine/cisplatin in patients with locally advanced or metastatic urothelial cancer without prior systemic therapy: EORTC Intergroup Study 30987. Journal of Clinical Oncology: Official Journal of the American Society of Clinical Oncology 2012;30(10):1107-13.

[9] Necchi A, Mariani L, Giannatempo P, Raggi D, Fare E, Nicolai $\mathrm{N}$, et al. Long-term efficacy and safety outcomes of modified (simplified) MVAC (methotrexate/vinblastine/doxorubicin/cisplatin) as frontline therapy for unresectable or metastatic urothelial cancer. Clinical Genitourinary Cancer 2014;12(3):203-9.e1.

[10] Heidenreich A, Wilop S, Pinkawa M, Porres D, Pfister D. Surgical resection of urological tumor metastases following medical treatment. Deutsches Arzteblatt International 2012;109(39):631-7.

[11] Pfannschmidt J, Hoffmann H, Dienemann H. Thoracic metastasectomy for nonseminomatous germ cell tumors. Journal of Thoracic Oncology: Official Publication of the International Association for the Study of Lung Cancer 2010;5(6 Suppl 2):S182-6.

[12] Rasco DW, Assikis V, Marshall F. Integrating metastasectomy in the management of advanced urological malignancies-where are we in 2005? The Journal of Urology 2006;176(5):1921-6.

[13] Tonyali S, Yazici S. Does solitary- and organ-confined metastasectomy really improve survival in advanced urologic malignancies? International Urology and Nephrology 2016;48(5):671-80.

[14] Dimopoulos MA, Finn L, Logothetis CJ. Pattern of failure and survival of patients with metastatic urothelial tumors relapsing after cis-platinum-based chemotherapy. The Journal of Urology 1994;151(3):598-600; discussion -1.

[15] Shamseer L, Moher D, Clarke M, Ghersi D, Liberati A, Petticrew M, et al. Preferred reporting items for systematic review and meta-analysis protocols (PRISMA-P) 2015: Elaboration and explanation. BMJ (Clinical Research ed) 2015;349:g7647.

[16] Higgins JGS. Cochrane Handbook for Systematic Reviews of Interventions Version 5.1.0. The Cochrane Collaboration. 2011;Available from: www.handbook.cochrane.org

[17] Tierney JF, Stewart LA, Ghersi D, Burdett S, Sydes MR Practical methods for incorporating summary time-to-event data into meta-analysis. Trials 2007;8:16.

[18] Anderson RS, el-Mahdi AM, Kuban DA, Higgins EM. Brain metastases from transitional cell carcinoma of urinary bladder. Urology 1992;39(1):17-20.

[19] Sweeney P, Millikan R, Donat M, Wood CG, Radtke AS, Pettaway CA, et al. Is there a therapeutic role for postchemotherapy retroperitoneal lymph node dissection in metastatic transitional cell carcinoma of the bladder? Journal of Urology 2003;169(6):2113-7.

[20] Otto T, Krege S, Suhr J, Rubben H. Impact of surgical resection of bladder cancer metastases refractory to systemic 
therapy on performance score: A phase II trial. Urology 2001;57(1):55-9.

[21] Abe T, Kitamura H, Obara W, Matsumura N, Tsukamoto $\mathrm{T}$, Fujioka $\mathrm{T}$, et al. Outcome of metastasectomy for urothelial carcinoma: A multi-institutional retrospective study in Japan. The Journal of urology 2014;191(4):932-6.

[22] Bekku K, Saika T, Kobayashi Y, Kioshimoto R, Kanbara T, Nasu Y, et al. Could salvage surgery after chemotherapy have clinical impact on cancer survival of patients with metastatic urothelial carcinoma? International Journal of Clinical Oncology 2013;18(1):110-5.

[23] Lehmann J, Suttmann H, Albers P, Volkmer B, Gschwend $\mathrm{JE}$, Fechner G, et al. Surgery for metastatic urothelial carcinoma with curative intent: The German experience (AUO AB 30/05). European Urology 2009;55(6):1293-9.

[24] Dodd PM, McCaffrey JA, Herr H, Mazumdar M, Bacik J, Higgins $\mathrm{G}$, et al. Outcome of postchemotherapy surgery after treatment with methotrexate, vinblastine, doxorubicin, and cisplatin in patients with unresectable or metastatic transitional cell carcinoma. Journal of Clinical Oncology: Official Journal of the American Society of Clinical Oncology 1999;17(8):2546-52.

[25] Siefker-Radtke AO, Walsh GL, Pisters LL, Shen Y, Swanson DA, Logothetis CJ, et al. Is there a role for surgery in the management of metastatic urothelial cancer? The M. D. Anderson experience. The Journal of Urology 2004;171(1):145-8.

[26] Matsuguma H, Yoshino I, Ito H, Goya T, Matsui Y, Nakajima $\mathrm{J}$, et al. Is there a role for pulmonary metastasectomy with a curative intent in patients with metastatic urinary transitional cell carcinoma? The Annals of Thoracic Surgery 2011;92(2):449-53.

[27] Necchi A, Giannatempo P, Lo Vullo S, Fare E, Raggi D, Nicolai N, et al. Postchemotherapy lymphadenectomy in patients with metastatic urothelial carcinoma: Long-term efficacy and implications for trial design. Clinical Genitourinary Cancer 2015;13(1):80-6.e1.

[28] Kim CJ, Wakabayashi Y, Kushima R, Sakano Y, Yoshiki T, Okada Y. Retroperitoneal lymph node dissection in patients with interaortocaval lymph node metastases of transitional cell carcinoma of the urinary tract. International Journal of Urology: Official Journal of the Japanese Urological Association 2004;11(4):243-7.

[29] Kim T, Ahn JH, You D, Jeong IG, Hong B, Hong JH, et al. Pulmonary Metastasectomy Could Prolong Overall Survival in Select Cases of Metastatic Urinary Tract Cancer. Clinical Genitourinary Cancer 2015;13(4):e297-304.

[30] Kanzaki R, Higashiyama M, Fujiwara A, Tokunaga T, Maeda J, Okami J, et al. Outcome of surgical resection of pulmonary metastasis from urinary tract transitional cell carcinoma. Interactive Cardiovascular and Thoracic Surgery 2010;11(1):60-4.

[31] Han WS, Kim K, Park JS. Result of surgical resection for pulmonary metastasis from urothelial carcinoma. The Korean Journal of Thoracic and Cardiovascular Surgery 2012;45(4):242-5.

[32] Cowles RS, Johnson DE, McMurtrey MJ. Long-term results following thoracotomy for metastatic bladder cancer. Urology 1982;20(4):390-2.

[33] Fokas E, Henzel M, Engenhart-Cabillic R. A comparison of radiotherapy with radiotherapy plus surgery for brain metastases from urinary bladder cancer: Analysis of 62 patients. Strahlentherapie und Onkologie: Organ der Deutschen Rontgengesellschaft [et al.] 2010;186(10): 565-71.

[34] Mahmoud-Ahmed AS, Suh JH, Kupelian PA, Klein EA, Peereboom DM, Dreicer R, et al. Brain metastases from bladder carcinoma: Presentation, treatment and survival. The Journal of Urology 2002;167(6):2419-22.

[35] Sundararajan S, Vogelzang NJ. Anti-PD-1 and PD-L1 therapy for bladder cancer: What is on the horizon? Future Oncology (London, England) 2015;11(16):2299-306.

[36] Rosenberg JE, Hoffman-Censits J, Powles T, van der Heijden MS, Balar AV, Necchi A, et al. Atezolizumab in patients with locally advanced and metastatic urothelial carcinoma who have progressed following treatment with platinumbased chemotherapy: A single-arm, multicentre, phase 2 trial. Lancet (London, England). 2016. 


\section{APPENDIX 1: EVIDENCE ACQUISITION SEARCH STRATEGIES}

\section{PubMed/MEDLINE}

((()((“carcinoma, transitional cell”'[MeSH Terms]) OR (Transitional Cell Carcinoma OR Transitional Cell Carcinomas OR urothelial carcinoma OR urothelial carcinomas))) AND (((((“Surgical Procedures, Operative"[Mesh]) OR "Radiotherapy”[Mesh]) OR “Metastasectomy”[Mesh])) OR ((Metastasectomy OR Metastasectomies OR surgical OR surgery OR radiotherapy)))) AND metastas*)) NOT ((“Animals”[Mesh]) NOT ((“Humans”[Mesh]) AND “Animals"[Mesh]))

\section{Embase (search via Ovid)}

1. transitional cell carcinoma/

2. (Transitional Cell Carcinoma or Transitional Cell Carcinomas or urothelial carcinoma or urothelial carcinomas).mp. [mp = title, abstract, subject headings, heading word, drug trade name, original title, device manufacturer, drug manufacturer, device trade name, keyword]
3. 1 or 2

4. surgery/

5. radiotherapy/

6. metastasis resection/

7. (Metastasectomy or Metastasectomies or surgical or surgery or radiotherapy).mp. [mp = title, abstract, subject headings, heading word, drug trade name, original title, device manufacturer, drug manufacturer, device trade name, keyword]

8. 4 or 5 or 6 or 7

9. metastas*.mp. [mp =title, abstract, subject headings, heading word, drug trade name, original title, device manufacturer, drug manufacturer, device trade name, keyword]

10. 3 and 8 and 9

11. animal/

12. human/

13. 11 and 12

14. 11 not 13

15. 10 not 14 